\title{
On Transmuted Type II Generalized Logistic Distribution with Application
}

\author{
Femi Samuel Adeyinka \\ Department of Mathematics, Obafemi Awolowo University, Ile-Ife, Nigeria
}

Email address:

fs.adeyinka247@gmail.com

\section{To cite this article:}

Femi Samuel Adeyinka. On Transmuted Type II Generalized Logistic Distribution with Application. American Journal of Applied Mathematics. Special Issue: On Transmuted Family of Distributions with Applications. Vol. 7, No. 6, 2019, pp. 177-182.

doi: 10.11648 j.ajam.20190706.15

Received: November 13, 2019; Accepted: December 17, 2019; Published: December 31, 2019

\begin{abstract}
Introducing extra parameters into the baseline distribution has been a huge breakthrough in research as this enhances more flexibility of the existing models. One of the recent methods is the use of transmutation map which has attracted the interest of many researchers in the last decade. This article investigates the flexibility of transmuted type II generalized logistic distribution. The well-known type II generalized logistic distribution is transmuted using quadratic rank transmutation map to develop a transmuted type II generalized logistic distribution. The map enables the introduction of additional parameter into its parent model to make it more flexible in the analysis of data in various disciplines such as biological sciences, actuarial science, finance and insurance. Some statistical properties of the model are considered and these properties include the moment, quantiles and functions of minimum and maximum order statistics. The estimation issue of the subject model is addressed using method of maximum likelihood estimation. The model is applied to real life data to demonstrate its performance and the comparison of the result of the subject model with its parent model was done using Akaike Information criterion (AIC), Corrected Akaike Information criterion (AICC) and Bayesian Information criterion (BIC) respectively. It is believed that the results from this research work will be of immense contributions in this field and other related disciplines in modelling real data.
\end{abstract}

Keywords: Generalized Logistic Distribution, Maximum Likelihood, Order Statistics, Parameter Estimation, Transmutatio

\section{Introduction}

Several probability models have been studied over the years with applications in different fields of endeavor which include biological science, actuarial science, clinical science and other related disciplines. Logistic distributions are probability models that are popularly used in modeling as normal distribution.

Its cumulative distribution function (cdf) in its simplest form is given by

$$
G(x)=\left(1+e^{-\left(\frac{x-\mu}{\sigma}\right)}\right)^{-1},-\infty<x<\infty
$$

with the location parameter $\mu \leq x$ and scale parameter $\sigma>$ 0 .

The model in (1) has gained generalizations by different authors which include Balakrishnan and Leung [1] which introduced shape parameter into the model and this has birthed type I generalized logistic distribution, type II generalized logistic distribution and type III generalized logistic distribution.

The transmutation of many baseline distributions has been studied by many authors in view of making them more flexible in analyzing data arising in various fields. Shaw and Beckley [2] developed a quadratic rank transmutation map. The map was applied to normal, exponential and uniform distribution respectively through which extra parameter was introduced. This map was applied to Gumbel distribution by Aryal [3], to weibul distribution by Aryal [4] and to the loglogistic distribution by Aryal [5]. Others that applied the same map to other probability models include Merovci et al [6] to some well-known distributions, Merovci et al [7] to Lindley-geometric distribution, Merovci et al [8] to generalized Rayleigh distribution, Merovci et al [9] to Pareto distribution, Merovci et al [10] to Lindley distribution, Adeyinka et al [11] to a generalized log-logistic distribution, Adeyinka et al [12] to a half logistic distribution, Adeyinka et al [13], Adeyinka [14] to logistic distribution, Adeyinka [15] to type I generalized logistic distribution. AL-Kadim et al 
[16] developed a cubic rank transmutation map and applied it to Weilbul distribution. Granzotto et al [17] suggested another cubic rank transmutation map and applied it to Weibul and log-logistic distributions. Rahman et al [18] obtained the general form of the work of Shaw et al [2].

This research article will focus on the transmuted form of type II generalized logistic distribution and its performance will be investigated with real data.

\section{Transmuted Type II Generalized Logistic Distribution}

Suppose a random variable $\mathrm{X}$ has the type II generalized logistic distribution with probability density function (pdf) and its cumulative distribution function (cdf) given by

$$
g(x)=\frac{b e^{-b x}}{\left(1+e^{-x}\right)^{b+1}},-\infty<x<\infty, b>0
$$

and

$$
G(x)=1-\frac{e^{-b x}}{\left(1+e^{-x}\right)^{b}},-\infty<x<\infty, \mathrm{b}>0
$$

respectively where $b>0$ is the shape parameter. The corresponding transmuted type II generalized logistic distribution, using the quadratic rank transmutation map suggested by Shaw et al [2],

$$
F(x)=(1+\lambda) G(x)-\lambda G^{2}(x),|\lambda| \leq 1
$$

is given by

$$
F(x)=\frac{\left(1+e^{-x}\right)^{b}\left\{\left(1+e^{-x}\right)^{b}-(1-\lambda) e^{-b x}\right\}-\lambda e^{-2 b x}}{\left(1+e^{-x}\right)^{2 b}},-\infty<x<\infty, b>0
$$

which is the cdf of transmuted type II generalized logistic distribution. The corresponding pdf is obtained by

differentiating (5) with respect to $x$ and it is given by

$$
f(x)=\frac{b e^{-b x}\left\{(1-\lambda)\left(1+e^{-x}\right)^{b}+2 \lambda e^{-b x}\right\}}{\left(1+e^{-x}\right)^{2 b+1}},-\infty<x<\infty, b>0
$$

with transmutation parameter $\lambda$. It is observed that when parameter $\lambda=0$ in (5) it gives the cdf of type II generalized logistic distribution in (3) and by setting $\lambda=0$ and $b=1.0$ respectively in (5) it gives the cdf of ordinary logistic

$b=2.0$
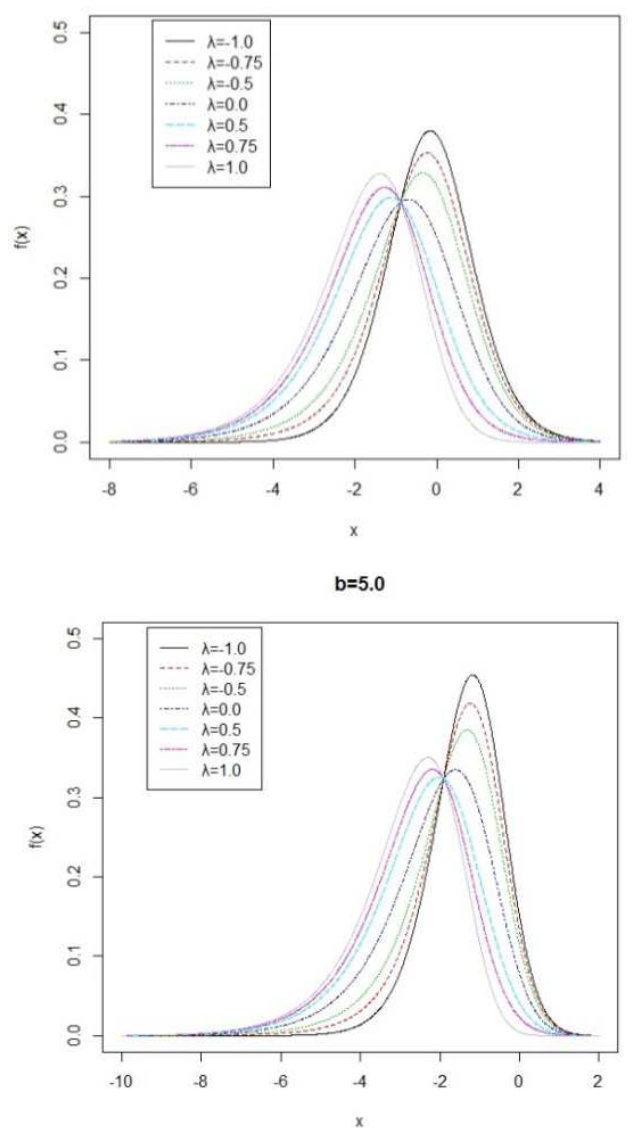

distribution (1) with $\mu=0$ and $\sigma=1$.

The pdf and cdf of the model are illustrated graphically in figures 1 and 2 for some selected values of parameters $b$ and $\lambda$ respectively.
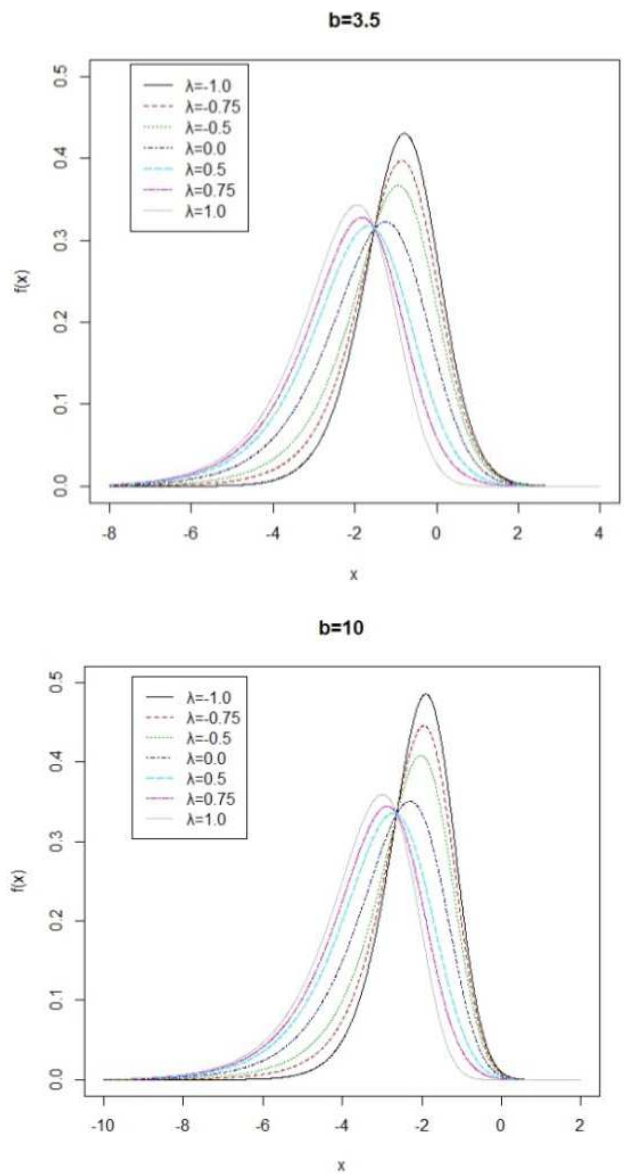

Figure 1. The probability distribution function of transmuted type II generalized logistic distribution. 

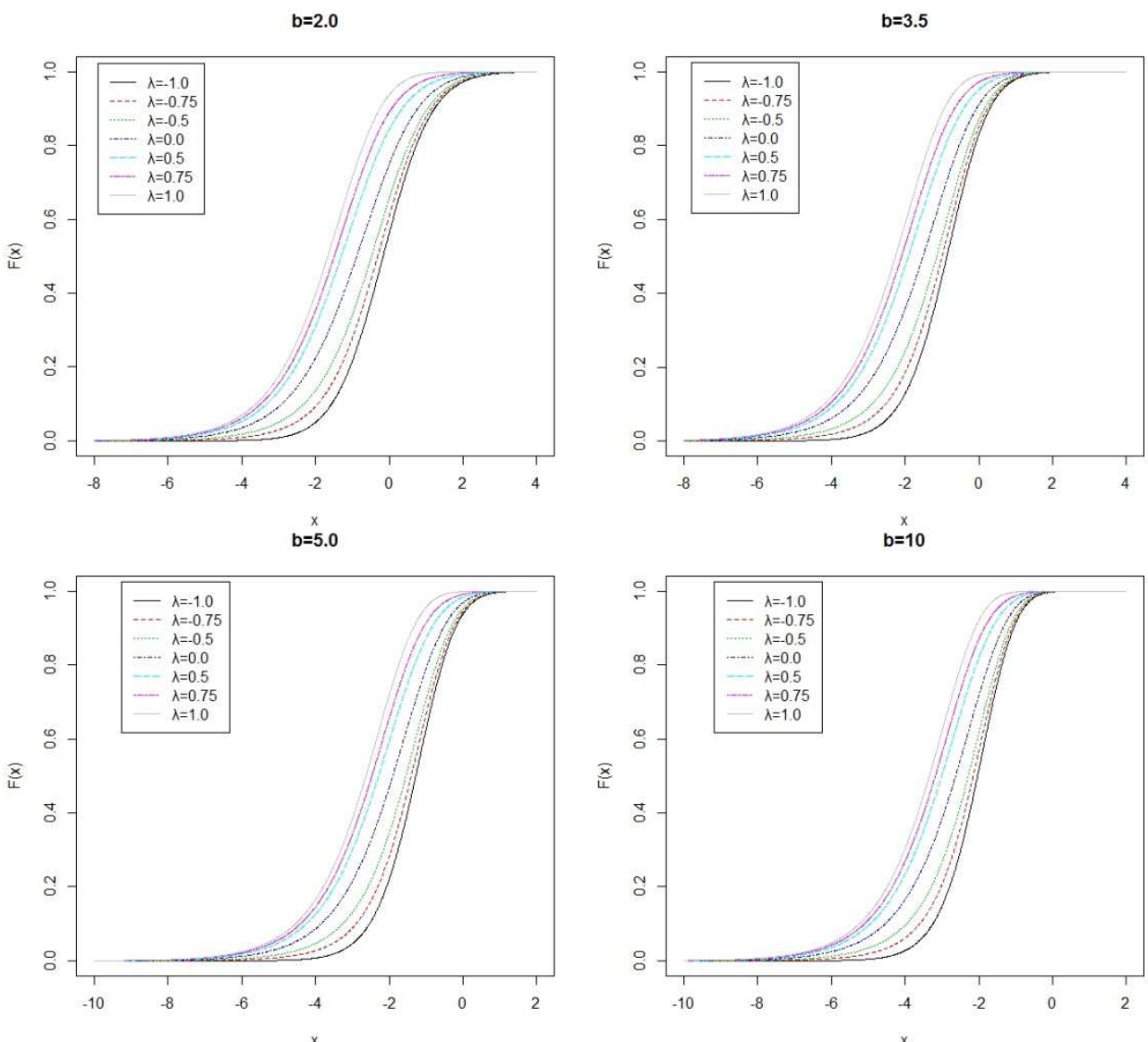

Figure 2. The cumulative distribution function of transmuted type II generalized logistic distribution.

\section{Moment Generating Function and Quantiles}

The moment generating function of a random variable, say $X$, is denoted by

$$
M_{X}(\mathrm{t})=\mathrm{E}\left[e^{t X}\right]=\int_{-\infty}^{\infty} e^{t x} f(x) d x .
$$

Suppose $f(x)$ is the pdf of transmuted type II generalized logistic distribution, on substitution, (7) becomes

$$
b\left[(1-\lambda) \int_{0}^{1}(1-u)^{b-t-1} u^{t} d u 2 \lambda \int_{0}^{1}(1-u)^{b-t-1} u^{b+t} d u\right]
$$

The integral part of (10) is equivalent to a beta function which can be written as

$B(a, b)=\int_{0}^{1} x^{a-1}(1-x)^{b-1} d x$. These relationships also hold

$$
B(a, b)=\frac{\Gamma(a) \Gamma(b)}{\Gamma(a+b)} \text { and } \Gamma(n)=(n-1) !
$$

By applying these (10) becomes

$$
(1-\lambda) \frac{\Gamma(b-t) \Gamma(1+t)}{\Gamma(b)}+\lambda \frac{\Gamma(b-t) \Gamma(b+t+1)}{\Gamma(2 b)}
$$

Similarly, the characteristic function of the transmuted type II generalized logistic distribution can be written as

$$
b\left[(1-\lambda) \int_{-\infty}^{\infty} \frac{e^{-(b-t) x}}{\left(1+e^{-x}\right)^{b+1}} d x+2 \lambda \int_{-\infty}^{\infty} \frac{e^{-(b-t) x}}{\left(1+e^{-x}\right)^{2 b+1}} d x\right]
$$

By taking $y=e^{-x}, x=-\ln y$ and $d x=-\frac{1}{y} d y$ in (8), on substitution it becomes

$$
b\left[(1-\lambda) \int_{0}^{\infty} \frac{y^{b-1-t}}{(1+y)^{b+1}} d y+2 \lambda \int_{0}^{\infty} \frac{y^{b-t}}{(1+y)^{2 b+1}} d y\right]
$$

If $u=\frac{1}{1+y}, y=\frac{1-u}{u}$ and $d y=-\frac{1}{u^{2}} d y$ in (9) then it gives

$$
Q_{X}(i t)=(1-\lambda) \frac{\Gamma(b-i t) \Gamma(1+i t)}{\Gamma(b)}+\lambda \frac{\Gamma(b-i t) \Gamma(b+i t+1)}{\Gamma(2 b)}
$$

Other properties such as mean, variance, skewness and kurtosis of the model can be obtained from (11).

The $q^{t h}$-quantile of the random variable $X$ is given by

$$
F_{X}\left(x_{q}\right)=q
$$

If $F_{X}(x)$ is the cdf of transmuted type II generalized logistic distribution (13) becomes

$$
\frac{\left(1+e^{-x} q\right) b\left\{\left(1+e^{-x} q\right)^{b}-(1-\lambda) e^{-b x} q\right\}-\lambda e^{-2 b x} q}{\left(1+e^{-x} q\right)^{2 b}}=q
$$


which on solving for $x_{q}$ in (14) gives

$$
x_{q}=\ln \left\{\left[\frac{(1-\lambda)+\sqrt{(1+\lambda)^{2}-4 \lambda q}}{2(1-q)}\right]^{1 / b}-1\right\}
$$

The median of the model is obtained by making $q=0.5$ in (15) which becomes

$$
x_{0.5}=\ln \left\{\left[(1-\lambda)+\sqrt{1+\lambda^{2}}\right]^{1 / b}-1\right\}
$$

\section{Random Number Generation}

By method of inversion random numbers can be generated from a given distribution with cdf given by

$$
F_{X}(x)=u
$$

where $u$ is uniformly distributed on $(0,1)$. Suppose $F_{X}(x)$ is the cdf of transmuted type II generalized logistic distribution, on substitution (17) becomes

$$
\frac{\left(1+e^{-x}\right)^{b}\left\{\left(1+e^{-x}\right)^{b}-(1-\lambda) e^{-b x}\right\}-\lambda e^{-2 b x}}{\left(1+e^{-x}\right)^{2 b}}=u .
$$

On solving for $x$ in (18) it becomes

$$
x=\ln \left\{\left[\frac{(1-\lambda)+\sqrt{(1+\lambda)^{2}-4 \lambda u}}{2(1-u)}\right]^{1 / b}-1\right\}
$$

For known values of parameters $b$ and $\lambda$ random numbers can be generated from (19).

\section{Order Statistics}

Given a random sample $X_{1}, X_{2} \ldots X_{n}$ from a continuous population with $\operatorname{cdf} F_{X}(x)$ and $\operatorname{pdf} f_{X}(x)$, the arrangement

$$
\begin{gathered}
X_{(1)} \leq X_{(2)} \leq \cdots \leq X_{(n)} \\
f_{X_{(r)}}(x)=\frac{b e^{-b(n-r+1) x}\left\{(1-\lambda)\left(1+e^{-x}\right)^{b}+2 \lambda e^{-b x}\right\}\left\{\left(1+e^{-x}\right)^{2 b}-(1-\lambda) e^{-b x}\left(1+e^{-x}\right)^{b}-\lambda e^{-2 b x}\right\}^{r-1}\left\{(1-\lambda)\left(1+e^{-x}\right)+\lambda e^{-b x}\right\}^{n-r}}{B(r, n-r+1)\left(1+e^{-x}\right)^{2 b n+1}}
\end{gathered}
$$

By setting $r=n$ in (26) we have the pdf of the largest observation $X_{(n)}$ which is given by

$$
f_{X_{(n)}}(x)=\frac{n b e^{-b n x}\left\{(1-\lambda)\left(1+e^{-x}\right)^{b}+2 \lambda e^{-b x}\right\}\left\{(1-\lambda)\left(1+e^{-x}\right)+\lambda e^{-b x}\right\}^{n-1}}{\left(1+e^{-x}\right)^{2 b n+1}}
$$

when taking $r=1$ in (26) we have the pdf of the smallest observation $X_{(1)}$ and it is given by

$$
f_{X_{(1)}}(x)=\frac{n b e^{-b x}\left\{(1-\lambda)\left(1+e^{-x}\right)^{b}+2 \lambda e^{-b x}\right\}\left\{\left(1+e^{-x}\right)^{2 b}-(1-\lambda) e^{-b x}\left(1+e^{-x}\right)^{b}-\lambda e^{-2 b x}\right\}^{n-1}}{\left(1+e^{-x}\right)^{2 b n+1}}
$$

\section{Estimation of Parameters}

Consider a sample $X_{1}, X_{2} \ldots X_{n}$ with sample size $n$ which is drawn from transmuted type II generalized logistic distribution whose pdf is given in (5). Its likelihood function is given by

$$
L=b^{n} e^{-b \sum_{i=1}^{n} x_{i}} \prod_{i=1}^{n}\left\{(1-\lambda)\left(1+e^{-x_{i}}\right)^{b}+2 \lambda e^{-b x_{i}}\right\} / \prod_{i=1}^{n}\left(1+e^{-x_{i}}\right)^{2 b+1} .
$$

By taking the natural logarithm of (29) it becomes

$$
\ln L=n \ln b-b \sum_{i=1}^{n} x_{i}-(2 b+1) \sum_{i=1}^{n} \ln \left(1+e^{-x_{i}}\right)+\sum_{i=1}^{n} \ln \left\{(1-\lambda)\left(1+e^{-x_{i}}\right)^{b}+2 \lambda e^{-b x_{i}}\right\} .
$$


Each estimate of the inherent parameters $b$ and $\lambda$ can be obtained by taking the derivative of (30) with respect to each parameter and equate the result to zero to obtain

$$
\begin{gathered}
\frac{\partial \ln L}{\partial \lambda}=\sum_{i=1}^{n} \frac{2 e^{-b x_{i}}-\left(1+e^{-x_{i}}\right)^{b}}{\left[(1-\lambda)\left(1+e^{-x_{i}}\right)^{b}+2 \lambda e^{-b x_{i}}\right]}=0 \\
\frac{\partial \ln L}{\partial b}=\frac{n}{b}-\sum_{i=1}^{n} x_{i}-2 \sum_{i=1}^{n} \ln \left(1+e^{-x_{i}}\right)-\sum_{i=1}^{n} \frac{2 \lambda x_{i} e^{-b x_{i}}-b(1-\lambda)\left(1+e^{-x_{i}}\right)^{b-1}}{\left[(1-\lambda)\left(1+e^{-x_{i}}\right)^{b}+2 \lambda e^{-b x_{i}}\right]}=0
\end{gathered}
$$

The system of equations obtained in (31) and (32) are nonlinear in parameter. Therefore, the maximum likelihood estimator $\hat{\vartheta}=(\widehat{b}, \hat{\lambda})^{\prime}$ of parameters $\vartheta=(b, \lambda)^{\prime}$ cannot be obtained analytically and as a result numerical method, quasi-Newton algorithm, is used to maximize the loglikelihood function in (30).

\section{Application}

This data is originally considered by Badar and Priest [21] and also used by Gupta et al [20]. It is the strength measured in GPA, for single carbon fibers and impregnated 1000carbon fiber tows. The fibers were tested under tension at gauge lengths of 1, 10, 20 and $50 \mathrm{~mm}$. Impregnated tows of 1000 fibers were tested at gauge lengths of 20, 50, 150 and $300 \mathrm{~mm}$. To illustrate this single fibers data set of $10 \mathrm{~mm}$ in gauge lengths with sample size 63 . The data are:

$1.901,2.132,2.203,2.228,2.257,2.350,2.361,2.396$, $2.397,2.445,2.454,2.474,2.518,2.522,2.525,2.532,2.575$, 2.614, 2.616, 2.618, 2.624, 2.659, 2.675, 2.738, 2.740, 2.856, 2.917, 2.928, 2.937, 2.937, 2.977, 2.996, 3.030, 3.125, 3.139, $3.145,3.220,3.223,3.235,3.243,3.264,3.272,3.294,3.332$, $3.346,3.377,3.408,3.435,3.493,3.501,3.537,3.554,3.562$,
$3.628,3.852,3.871,3.886,3.971,4.024,4.027,4.225,4.395$, 5.020 .

Using quasi Newton algorithm in R package, the results of the analysis are shown in Table 1 . The test of the performance of each model is done with Akaike Information criterion (AIC), Corrected Akaike Information criterion (AICC) and Bayesian Information criterion (BIC) respectively. The results show that the transmuted type II generalized logistic distribution (TGLD) has a better goodness of fit than its parent model (GLD) which is given in (2). The following are defined as

$$
\begin{gathered}
A I C=2 k-2 L L \\
A I C C=A I C+\frac{2 k(k+1)}{n-k-1}
\end{gathered}
$$

and

$$
B I C=2 \log (n)-2 L L
$$

with $\mathrm{k}$ given as the number of parameters in the model and $\mathrm{n}$ as the sample size and LL as the maximized value of $\log$ likelihood function.

Table 1. Outcomes of the analysis on the performance of the models.

\begin{tabular}{lllll}
\hline Model & Estimates & -LL & AIC & AICC \\
\hline TGLD & $\hat{b}=2.570 \hat{\lambda}=0.427$ & 114.352 & 232.704 & 232.904 \\
GLD & $\hat{b}=0.552$ & 121.126 & 244.252 & 244.318 \\
\hline
\end{tabular}

\section{Conclusion}

A new probability model has emerged from the type II generalized logistic model and this is called transmuted type II generalized logistic distribution. It has been demonstrated that the model is more flexible than its parent model. The mathematical properties which include moment generating function, quantiles, pdfs of the smallest and largest observation and characteristic function of the model are established and the estimation of parameters inherent in the subject model is properly considered. A real life data is used to illustrate the applicability of the model and compared the performance to its parent model. This model is recommended in the analysis of data in various disciplines where the real data follow this model.

\section{References}

[1] Balakrishnan N. and Leung M. Y. (1988). Order Statistics from the Type I Generalized logistic distribution. Communications in Statistics simulation and computation. Vol 17 (1) 25-50.
[2] Shaw, W. T, and Buckley, I. R. (2009). Alchemy of Probability Distributions: Beyond Gram-Charlier and Cornish -Fisher Expansions, and Skewed- kurtotic Normal Distribution from a Rank Transmutation Map. arxivpreprint arxiv: 0901.0434 .

[3] Aryal, G. R, and Tsokos, C. P. (2009). On the transmuted extreme value distribution with application. Nonlinear Analysis: Theory, Methods and Application.71 (12), el401el407.

[4] Aryal, G. R, and Tsokos, C. P. (2011). Transmuted Weilbull distribution: A generalization of Weilbull probability distribution. European Journal of Pure and Applied Mathematics. 4 (2), 89-102.

[5] Aryal, G. R. (2013). Transmuted log-logistic distribution. Journal of Statistics Applications and probability. 2 (1), 11-20.

[6] Merovci, F., Alizadeh, M., and Hamedani, G. (2016). Another Generalized Transmuted Family of Distributions: Properties and Applications. Austrian Journal of Statistics. 45, 71-93.

[7] Merovci, F., Elbatal, I. (2014). Transmuted Lindley-geometric Distribution and its Applications. Journal of Statistics Applications and Probability. 3 (1), 77-91. 
[8] Merovci, F. (2014). Transmuted Generalized Rayleigh Distribution. Journal of Statistics Applications and Probability. $3(1), 9-20$.

[9] Merovci, F., Puka, L. (2014). Transmuted Pareto Distribution. Probstat.7, 1-11.

[10] Merovci, F. (2013). Transmuted Lindley Distribution. International Journal of open Problems in Computer Science and Mathematics. 6 (2), 63-72.

[11] Adeyinka F. S, and Olapade, A. K. (2019). On Transmuted Four Parameters Generalized Log-Logistic Distribution. International Journal of Statistical Distributions and Applications. 5 (2): 32-37.

[12] Adeyinka F. S, and Olapade A. K. (2019). A Study on Transmuted Half Logistic Distribution: Properties and Application. International Journal of Statistical Distributions and Applications. 5 (3): 54-59.

[13] Adeyinka F. S, and Olapade, A. K. (2019). On the Flexibility of a Transmuted Type I Generalized Half-Logistic Distribution with Application. Engineering Mathematics. 3 (1): 13-18.

[14] Adeyinka F. S. (2019). On the Performance of Transmuted Logistic Distribution: Statistical Properties and Application. Budapest International Research in Exact Sciences (BirEx) Journal. 1 (3): 34-42.
[15] Adeyinka, F. S. (2019). On the Tractability of Transmuted Type I Generalized Logistic Distribution with Application. International Journal of Theoretical and Applied Mathematics. 5 (2): $31-36$.

[16] AL-Kadim, K. A. and Mohammed, M. H. (2017). The cubic transmuted Weibull distribution. Journal of University of Babylon, 3: 862876 .

[17] Granzotto, D. C. T., Louzada, F., and Balakrishnan, N. (2017). Cubic rank transmuted distributions: Inferential issues and applications. Journal of statistical Computation and Simulation.

[18] Rahman M. M, Al-Zahrani B, Shahbaz M. Q (2018). A general transmuted family of distributions. Pak J Stat Oper Res 14: 451-469.

[19] David, H. A. (1970) Order Statistics. New York: Wiley Interscience series.

[20] Badar, M. G. and Priest, A. M. (1982), "Statistical aspects of fiber and bundle strength in hybrid composites", Progress in Science and Engineering Composites, Hayashi, T., Kawata, K. and Umekawa, S. (eds.), ICCM-IV, Tokyo, 1129-1136.

[21] Gupta, R. D., Kundu, D. (2010). Generalized Logistic Distributions. Journal of Applied Statistical Science.18, 51-66. 